\title{
HISTOLOGICAL EVALUATION OF THE ANTIOXIDANT EFFECT OF VITAMIN E ON REVERSING THE NEGATIVE IMPACT OF TARTRAZINE ON EXTRACTION SOCKET HEALING. (RANDOMIZED CONTROLLED TRIAL)
}

\author{
Raneem F. Obeid ${ }^{*}$, Hesham S. Abdelmoneim ${ }^{* *}$ and Radwa T. Elsharkawy ${ }^{* * *}$
}

\begin{abstract}
One of a synthetic food dye which used worldwide is Tartrazine, it is an orange-colored, water soluble with a potential toxicological risk. Food or drinks containing tartrazine caused sensitivity symptoms. Vitamin E is an essential lipid-soluble vitamin and has well-established antioxidant properties which improve the proliferation, differentiation, and mineralization, and reduced apoptosis of osteoblastic cells.
\end{abstract}

Aim of this study: to evaluate the effect of tartrazine and co-administration of vitamin $\mathrm{E}$ on post extraction socket using histological examination by light microscope.

Materials and methods: Surgical extraction of mandibular first molars were done in a twenty one albino rats, the animals were randomly divided into 3 groups; group I (control), group II given tartrazine and group III given tartrazine and Vitamin E (treated group). The duration of the experiments was 14 days. Histological evaluation was done by H \& E and Masson trichrome.

Results: the bone healing of the extraction socket in the Vitamin E treated group was nearly the same as the control group.

Conclusion: Vitamin $\mathrm{E}$ has a protective effect as an effective antioxidant on bone healing in post extraction socket and may be useful as a supportive agent in an endless array of dental surgeries.

KEYWORDS: Extraction, Socket, Vitamin E, Tartrazine, Antioxidant, food color, bone healing.

* Lecturer of Oral Biology, Faculty of Oral and Dental Medicine, Future University in Egypt.

** Lecturer of Oral and Maxillofacial Surgery, Faculty of Dentistry, Cairo University

*** Lecturer of Oral Biology, Faculty of Oral and Dental Medicine, Future University in Egypt. 


\section{INTRODUCTION}

Natural colors are more expensive, less stable and possess low color intensity, while synthetic colors are more stable, impart better color, uniformity and blend together easily to provide a wide range of hues. The random use of food colors poses a serious health hazards in human beings (Rezaei et al., 2015). One synthetic food dye used worldwide is Tartrazine. It is orange-colored, water soluble with a potential toxicological risk. It is used to color foodstuff such as soft drinks, juices, biscuits, ice creams, sauces, mustard, snacks, flavored processed cheese, as well as drugs and cosmetics (shampoos, colognes, toothpastes, soaps) (Mehedi et al., 2009)

(Rus et al., 2010) stated that food or drinks containing tartrazine made sensitivity symptoms. After ingestion of tartrazine humans develop anxiety, headaches, blurred vision, itching, general weakness, heat waves, feeling of suffocation, purple skin patches and sleep disorders.

The absorbed tartrazine is secreted in urine while, the remaining tartrazine is metabolized by intestinal microflora. The metabolite of tartrazine can generate reactive oxygen species (ROS), which in turn accelerate the oxidative stress, making a reduction of $\mathrm{O} 2$ that damage most cellular components, leading to cell death (Visweswaran \& Krishnamoorthy, 2012).

Antioxidant defense mechanisms, which may be enzymatic or non-enzymatic can eliminate the effect of free oxygen radical produced by tartrazine (Bansal et al., 2005).

Vitamin E is an essential lipid-soluble vitamin that consists of two subgroups: tocopherol and tocotrienol (Wong et al., 2019). Due to presence of tocotrienol, there is an improve in proliferation, differentiation, and mineralization, and reduced apoptosis of osteoblastic cells (Abd Manan, Mohamed, \& Shuid, 2012)and (Xu et al., 2018).

After tooth extraction, bone formation plays an important role in alveolar socket wound healing.
The metabolism of bone tissue depends on maintaining a delicate balance between bone resorption by osteoclasts and bone formation by osteoblasts. (Dias-da-Silva et al., 2013)

During the healing process, free radicals are generated that may impair healing, which has been associated with increased oxidative stress. Antioxidants, such as vitamin E, may therefore facilitate fracture healing by scavenging the free radicals and relieving oxidative stress. (Mohamad et al., 2012)

Healing through dietary strategies is an attractive option for patients, such that healing from delicate oral surgeries occurs as optimally as possible with minimal patient-meditated complications through food choices (Lau et al., 2013). The healing process in the animal model tends to be faster; therefore, there may be variation in the time frames discussed when using the data to describe the sequence of events post-extraction in humans (Morjaria, Wilson, \& Palmer, 2014).

So, the aim of this study is to evaluate the effect of tartrazine and co-administration of Vitamin $\mathrm{E}$ on post extraction socket using histological examination by light microscope.

\section{MATERIALS AND METHODS:}

\section{Materials:}

\section{Animals:}

Twenty-one adult male albino rats weighing between 200- 250 grams were used in this experiment. The experiment was carried out under the supervision of a specialized veterinarian at the Medical Research Center, Faculty of Medicine, Ain Shams University. Adequate medication and postoperative care were provided following surgical procedures. Sacrificed bodies were disposed of by the incinerator of Ain Shams University. The Research Ethics Committee of Ain Shams University approved the experimental procedure. 


\section{Drugs:}

a- Tartrazine powder was purchased from SIGMA ALDRICH $^{\circledR}$ (St. Louis, Missouri, United States) and given daily at a dose of $7.5 \mathrm{mg} / \mathrm{kg}$ in $1 \mathrm{ml}$ distilled water (Himri et al., 2011).

b- Oral supplementation of vitamin E capsules was given daily at a dose of $400 \mathrm{IU} / \mathrm{kg}$ vitamin dissolved in $1 \mathrm{ml}$ olive oil (Noaparast and Hosseinimehr 2013).

\section{Methods:}

\section{Experimental Design:}

Surgical extraction of mandibular first molars was done in all rats. Rats were randomly divided into 3 groups as listed in Table 1. Sacrifice was carried out after 14 days (Olaitan et al., 2019).

TABLE (1) Experimental groups description:

\begin{tabular}{|l|l|}
\hline Group I & Given $1 \mathrm{ml}$ distilled water. \\
\hline Group II & Given tartrazine in $1 \mathrm{ml}$ distilled water. \\
\hline Group III & $\begin{array}{l}\text { Given tartrazine in } 1 \mathrm{ml} \text { distilled water plus } \\
\text { vitamin } \mathrm{E} \text { in } 1 \mathrm{ml} \text { olive oil. }\end{array}$ \\
\hline
\end{tabular}

\section{Dental surgical procedure:}

All animals were fasted twelve hours before the operation to avoid aspiration of gastric contents during general anesthesia. Extraction was performed under sterile conditions. The animals were anesthetized by intraperitoneal administration of a 4:1 solution of ketamine/xylazine at a dose of $0.15 \mathrm{ml}$ per $100 \mathrm{~g}$ of body weight. The mandibular left first molar of rats was extracted atraumatically. Extraction was performed using a curved hemostat with a gentle buccolingual movement (Mahmoud \& Hegazy, 2013).

\section{Postoperative Care:}

i) Antibiotic: a single dose of 30,000 IU penicillin-G benzathin was administered intramuscularly to prevent infection. ii) Corticosteroid: $1 \mathrm{ml}$ of methyl prednisolone acetate (Depomedrol 40mg/ml, Egyptian International pharmaceutical) intramuscularly to reduce postoperative edema

iii) Analgesic and anti-inflammatory: Diclofenac Sodium (Voltaren $75 \mathrm{mg} / 3 \mathrm{ml}$, Novartis) intramuscularly to reduce postoperative pain.

During the first week after tooth extraction, animals were fed a soft food to avoid post-surgery trauma (Mahmoud \& Hegazy, 2013). The diet consisted of shredded fresh vegetables and soft bread. The rats were sacrificed by an overdose of anesthesia (thiopental sodium 40mg/kg) 14 days after tooth extraction (Olaitan et al., 2019).

\section{Preparation of specimens:}

The molar area of both jaw sides was excised free, after trimming the incisors area to facilitate penetration of the fixative and decalcifying agent. Specimens were fixed in $10 \%$ buffered formaldehyde for 24 hours. The specimens were decalcified using a solution containing $12 \%$ EDTA buffered in pH7.2 PBS for 30 days at $4^{\circ} \mathrm{C}$ (Bancroft \& Gamble, 2008). After complete decalcification, specimens were processed, infiltrated in paraffin wax and embedded in the center of wax blocks. The embedded specimens were cut into 5 microns thick sections. Specimens were sectioned and were stained by:

\section{Hematoxylin and Eosin $(H \& E)$ stain:}

After fixation, specimens were washed properly under running water, dehydrated by transferring through ascending concentrations of alcohol. The sections were stained by Hematoxylin and Eosin (H\&E) stain to be examined by light microscope (Trinocular microscope Olympus, BX46).

\section{Masson trichrome special stain:}

It was used to detect areas of new bone formation as green color while old bone areas without new collagen formation appear red- dish in color when 
examined by light microscope. Paraffin sections were fastened to slides with Masson's gelatin (Bancroft \& Gamble, 2002).

\section{RESULTS}

\section{Hematoxylin and Eosin (H\&E) stain}

Group I: The histological examination of the extracted sockets of this group after 14 days showed newly formed bone trabeculae partially filling the socket. These trabeculae lined by osteoblasts. Osteocytes appeared unorganized. They also showed a lot of resting as well as reversal lines. The connective tissues surrounding the newly formed bone matrix appeared rich in active fibroblasts, collagen fibers, undifferentiated mesenchymal cells and highly vascular blood vessels. (Fig 1 A)

Group II: The sections of group II showed thin trabecular bone strands, osteoblasts appeared dispersed in some areas and small number of osteocytes occupying relatively large lacunae. The connective tissue revealed an intense inflammatory cell infiltrate, large amounts of fibroblasts and immature collagen fiber bundles and extravasated RBCs. Some strands showed deep basophilic resting lines. (Fig 2A)

Group III: The sections of Group III treated with vitamin E showed trabecular bone strands lined by osteoblasts, less inflammatory cells infiltrate in the connective tissue, a lot of active fibroblasts and mature collagen fiber bundles, highly vascular blood vessels and no signs of RBCs extravasation.

(Fig 3A)

\section{Masson trichrome stain}

The Masson trichrome sections of group I showed large areas of immature bone matrix which stained green and small isolated areas within the matrix appeared red. The collagen bundles inside the bone marrow appeared green (Fig 1B). In group II, the sections showed dispersed immature collagen fibers in the connective tissue, the newly formed bone trabecular appeared also green (Fig 2B). The sections of the treated group III showed green trabecular bone matrix. The collagen bundles appeared green and more organized (Fig 3B).

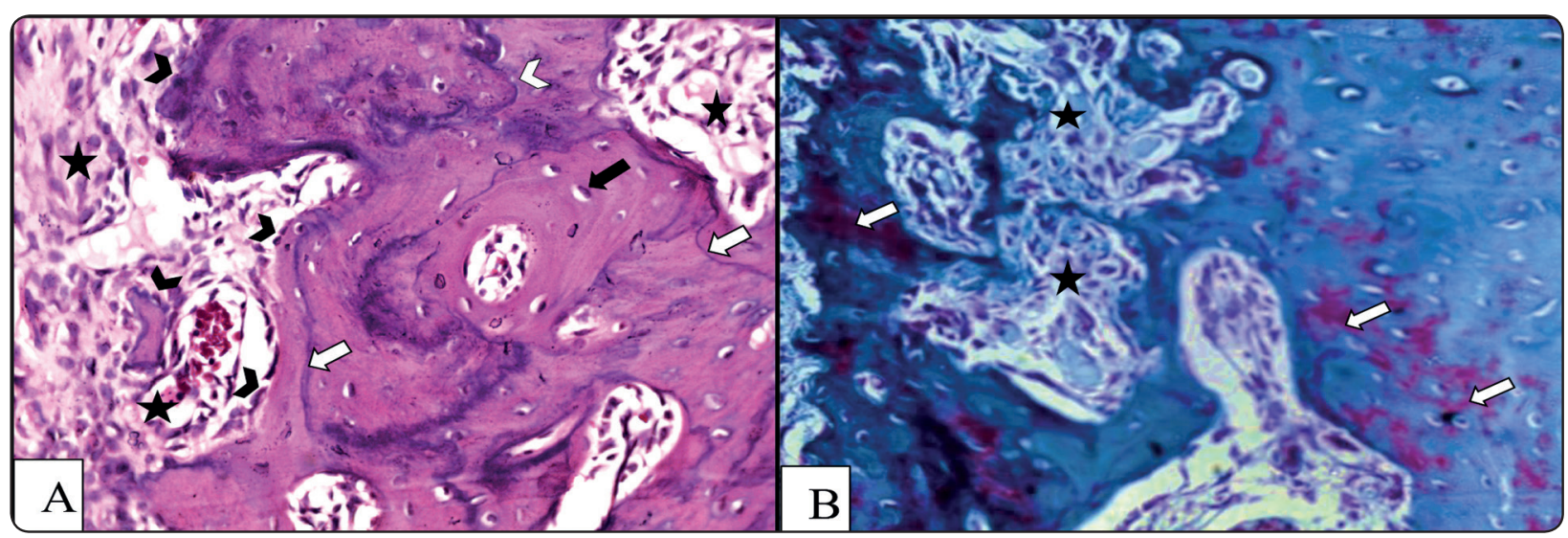

Fig 1A: Photomicrograph of the control group showing osteoblasts lining the newly formed bone trabeculae (arrow heads), osteocytes (black arrows), resting (white arrows) and reversal lines (white arrow head). Fibroblasts, collagen fibers, undifferentiated mesenchymal cells and highly vascular blood vessels in the connective tissue (black stars).H\&Ex400.

Fig 1B: Photomicrograph of the Masson trichrome stained section of the same group showing the collagen bundles inside the bone marrow (black stars), some mature areas of bone matrix within the bone trabeculae (white arrows). Masson trichrome X400. 


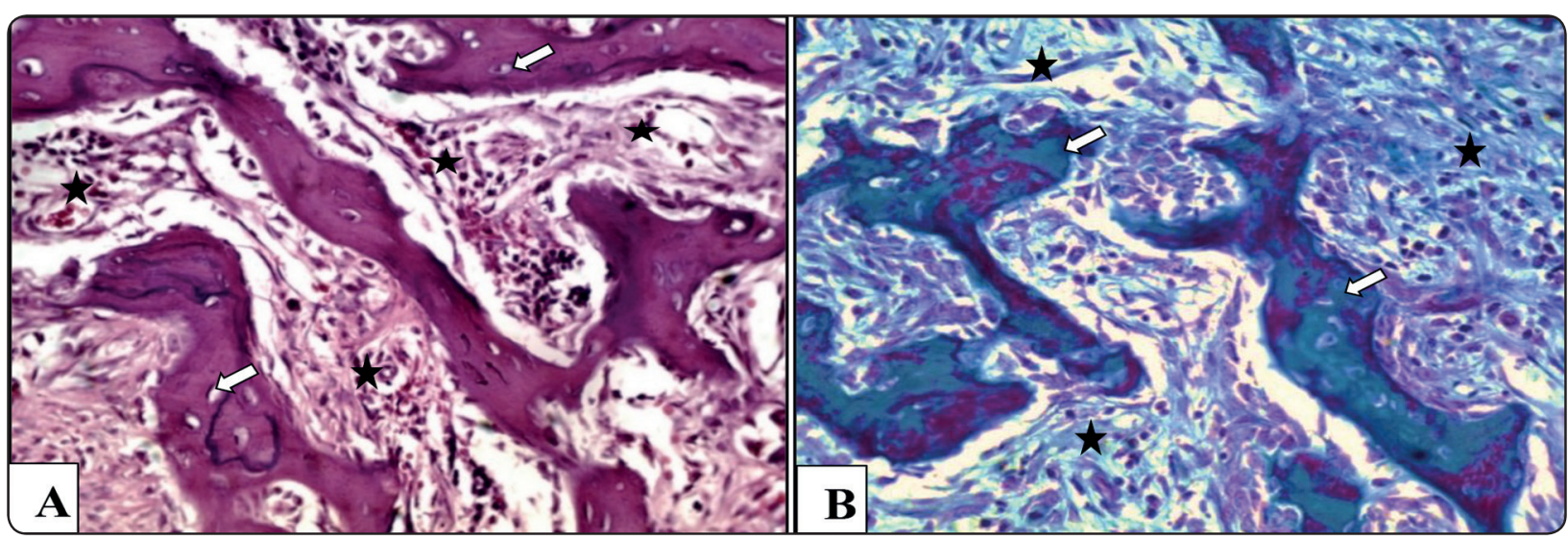

Fig 2A: Photomicrograph of group II showing thin trabecular bone strands, osteocytes occupying relatively large lacunae (white arrows), inflammatory cell infiltrate and extravasated RBCs (black stars). H\&Ex400.

Fig 2B: Photomicrograph showing large amounts of fibroblasts and dispersed immature collagen fiber bundles (black stars)and thin strands of newly formed trabeculae with dispersed mature areas(white arrows). Masson trichrome X400.
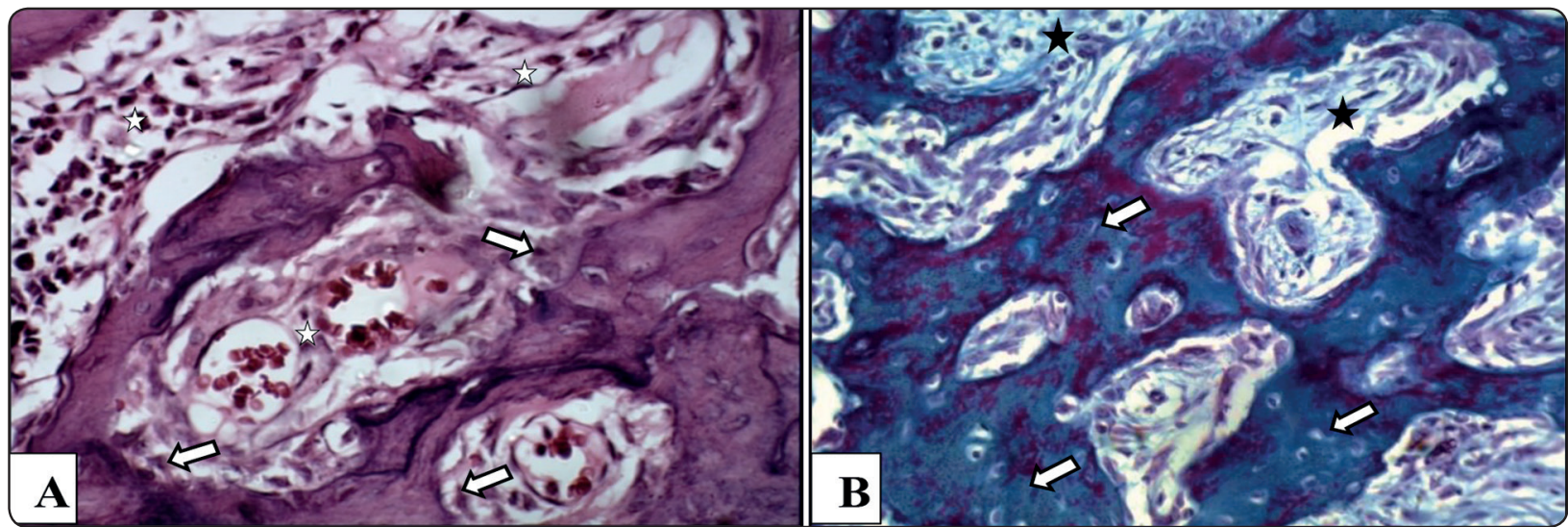

Fig 3A: Photomicrograph of group III showing the trabecular bone strands lined by osteoblasts (white arrows), less inflammatory cells, fibroblasts and mature collagen fiber bundles, highly vascular blood vessels (white stars). H\&Ex400

Fig 3B: Photomicrograph showing green trabecular bone matrix (white arrows). The collagen bundles appeared green and more organized(black stars). Masson trichrome X400.

\section{DISCUSSION}

Colors additives are generally used in various foods, including soft drinks, dairy products, grains, nuts, and ice cream. Although their application is strictly controlled in many countries it has been reported that some colors are harmful and induce adverse side effects (Amin, Hameid II \& Elsttar, 2010).

The purpose of this study was to evaluate the effect of tartrazine as a commonly used synthetic color additive on bone healing post extraction socket and the administration of Vitamin $\mathrm{E}$ as an effective antioxidant. That was done by histological examination of the bone healing in the socket of mandibular $1^{\text {st }}$ molar of albino rats after 14 days post extraction using the light microscope.

The preservation of sufficient bone volume with optimal bone quality and early healing of alveolar bone after tooth extraction are obviously favorable for subsequent dental treatment as implant dentistry. (Machida et al., 2010) stated that post-extraction healing in human began at the end of the first week; 
the socket became filled with granulation tissue that would be replaced with immature bone by eight weeks. However, rat extraction sockets filled gradually by newly-formed bone starting from 2 weeks as it is a non-critical size defect (Cohen \& Cohen-Lévy, 2014).

The healing process exhibited successive and overlapping events; started by blood clot formation, proliferation of mesenchymal cells, granulation tissue formation with inflammatory cells infiltration, angiogenesis, proliferation of fibroblasts and collagen synthesis; bone formation and finally bone remodeling (Vieira et al., 2015).

In the present study, the extraction sockets of rats of group II given tartrazine showed delayed bone healing in comparison with the control group and the vitamin $E$ treated group at the end of the second week, that was demonstrated by the amount of formed bone trabeculae and their degree of maturation which were less when compared to the control group.

The consumption of tartrazine stimulates the processes of lipid peroxidation and inhibits the activity of antioxidant system resulting in the formation of free radicals, so induce oxidative stresses and cause damage to cell membranes, proteins, nuclear chromatin and instability of ionic channels and receptors (Elhkim et al., 2007) (Visweswaran \& Krishnamoorthy, 2012), that might explain the distortion of the osteoblastic activity in some areas in group II of this study. The oxidative stress is due to the disruption of the equilibrium between production of free radicals and their being scavenged by the antioxidant system.

In group II, the intense inflammatory cell infiltration in the connective tissue, immature collagen fiber bundles and extravasated RBCs signify the delay in the bone healing events which were highly influenced by the increase of these free radicals, this came in accordance with (Mohamad et al., 2012) where they stated that during the healing process, free radicals are generated that may impair healing, which has been associated with increased oxidative stress.

The results of the present research revealed that control group and the vitamin E treated group had an increase in the amount of newly formed bone trabeculae, large amounts of fibroblasts and mature collagen bundles, less inflammatory cells infiltration and no signs of RBCs extravasation. This came with agreement of the normal socket bone healing events (Vieira et al., 2015).

Vitamin E had a well-established antioxidant property. Its administration reduced lipid peroxidation; this might be related to high lipid solubility of vitamin $\mathrm{E}$ which facilitate its free distribution in the cell membrane. Vitamin E protects against damage induced by free radical and also reactive products of lipid peroxidation. Both isoforms of vitamin $\mathrm{E}$, tocopherol and tocotrienol scavenge the free radicals (Maniam et al., 2008) and(Takhshid et al., 2012).

In addition, (Hasan et al.2018) demonstrated that tocotrienol enhanced osteogenic activity of osteoblasts by promoting the expression of bone formation-related genes, so there was increased in the formation of collagen fibrils and mineralization of the extracellular matrix.

From the analysis of the results of our study in addition to the reported benefits of Vitamin E, we could postulate the near infinite applications and advantages of Vitamin $\mathrm{E}$ administration to promote and enhance wound healing in the oral cavity, particularly secondary to the critical and sensitive surgical procedures which sometimes fail without a markable cause and where patients diet could be thought of now as the unexpected etiology. Thereby enhancing both soft and hard tissue healing in the oral cavity could benefit a large array of procedures ranging from simple extraction procedures, to surgical extractions, to dental implant surgeries, to various bone grafting procedures, to large defects reconstruction, to orthognathic surgeries and a lot more. 


\section{CONCLUSION}

Vitamin E has a significant role as an effective antioxidant on promoting bone healing in post extraction sockets and may be considered as a useful and supportive agent in an endless array of dental surgical applications.

\section{REFERENCES}

- $\quad$ Abd Manan, N., Mohamed, N., \& Shuid, A. N. (2012). Effects of low-dose versus high-dose $\gamma$-tocotrienol on the bone cells exposed to the hydrogen peroxide-induced oxidative stress and apoptosis. Evidence-Based Complementary and Alternative Medicine, 2012.

- $\quad$ Amin, K., Hameid II, H. A., \& Elsttar, A. A. (2010). Effect of food azo dyes tartrazine and carmoisine on biochemical parameters related to renal, hepatic function and oxidative stress biomarkers in young male rats. Food and Chemical Toxicology, 48(10), 2994-2999.

- $\quad$ Bancroft, J., \& Gamble, M. (2002). Theory and Practice of Histological Techniques 5th Edition, eds. Bancroft, JD and Gamble, M., Churchill Livingstone.

- Bancroft, J. D., \& Gamble, M. (2008). Theory and practice of histological techniques: Elsevier health sciences.

- $\quad$ Bansal, A., Bansal, M., Soni, G., \& Bhatnagar, D. (2005). Modulation of N-nitrosodiethylamine (NDEA) induced oxidative stress by vitamin $\mathrm{E}$ in rat erythrocytes. Human \& experimental toxicology, 24(6), 297-302.

- Cohen, N., \& Cohen-Lévy, J. (2014). Healing processes following tooth extraction in orthodontic cases. Journal of Dentofacial Anomalies and Orthodontics, 17(3).

- Dias-da-Silva, M. A., Pereira, A. C., Marin, M. C., \& Salgado, M. A. (2013). The influence of topic and systemic administration of copaiba oil on the alveolar wound healing after tooth extraction in rats. Journal of clinical and experimental dentistry, 5(4), e169.

- $\quad$ Elhkim, M. O., Héraud, F., Bemrah, N., Gauchard, F., Lorino, T., Lambré, C., Poul, J.-M. (2007). New considerations regarding the risk assessment on Tartrazine: an update toxicological assessment, intolerance reactions and maximum theoretical daily intake in France. Regulatory Toxicology and Pharmacology, 47(3), 308-316.

- Hasan, W. N. W., Ghafar, N. A., Chin, K.-Y., \& Ima-Nirwana, S. (2018). Annatto-derived tocotrienol stimulates osteogenic activity in preosteoblastic MC3T3-E1 cells: A temporal sequential study. Drug design, development and therapy, 12, 1715 .

- Himri, I., Bellahcen, S., Souna, F., Belmekki, F., Aziz, M., Bnouham, M., Saalaoui, E. (2011). A 90-day oral toxicity study of tartrazine, a synthetic food dye, in wistar rats. Group, 300(00).

- $\quad$ Lau, B. Y., Johnston, B. D., Fritz, P. C., \& Ward, W. E. (2013). Dietary strategies to optimize wound healing after periodontal and dental implant surgery: an evidence-based review. The open dentistry journal, 7,36 .

- $\quad$ Machida, T., Nyan, M., Kon, K., Maruo, K., Sato, H., \& Kasugai, S. (2010). Effect of hydroxyapatite fiber material on rat incisor socket healing. Journal of Oral Tissue Engineering, 7(3), 153-162.

- Mahmoud,E.F., \& Hegazy, R. H. (2013). Evaluation of the Role of Bone Marrow-Derived Mesenchymal Stem Cells in Bone Regeneration of Dental Socket in StreptozotocinInduced Diabetic Albino Rats. Life Science Journal, 10(2).

- Maniam, S., Mohamed, N., Shuid, A. N., \& Soelaiman, I. N. (2008). Palm tocotrienol exerted better antioxidant activities in bone than $\alpha$-tocopherol. Basic \& clinical pharmacology \& toxicology, 103(1), 55-60.

- Mehedi, N., Ainad-Tabet, S., Mokrane, N., Addou, S., Zaoui, C., Kheroua, O., \& Saidi, D. (2009). Reproductive toxicology of tartrazine (FD and C Yellow No. 5) in Swiss albino mice. American Journal of Pharmacology and Toxicology, 4(4), 130-135.

- Mohamad, S., Shuid, A. N., Mohamed, N., Fadzilah, F. M., Mokhtar, S. A., Abdullah, S., Soelaiman, I. N. (2012). The effects of alpha-tocopherol supplementation on fracture healing in a postmenopausal osteoporotic rat model. Clinics, 67(9), 1077-1085.

- $\quad$ Morjaria, K. R., Wilson, R., \& Palmer, R. M. (2014). Bone healing after tooth extraction with or without an intervention: a systematic review of randomized controlled trials. Clinical implant dentistry and related research, 16(1), 1-20.

- $\quad$ Noaparast, Z. and S. J. Hosseinimehr (2013). "Radioprotective agents for the prevention of side effects induced by radioiodine-131 therapy." Future Oncology 9(8): 11451159 .

- Olaitan, O., et al. (2019). "Histologic assessment of extraction sockets following tooth extraction: Suitability of a rabbit model." Nigerian Journal of Dental Research 4(1): 1-4. 
- $\quad$ Rezaei, M., Abadi, F. S., Sharifi, Z., Karimi, F., Alimohammadi, M., Abadi, R. A. S., \& Roostaei, H. (2015). Assessment of synthetic dyes in food stuffs produced in confectioneries and restaurants in Arak, Iran. Thrita, 4(1).

- $\quad$ Rus, V., Gherman, C., Miclăuş, V., Mihalca, A., \& Nadăş, G. (2010). Comparative toxicity of food dyes on liver and kidney in guinea pigs: A histopathological study. Annals of the Romanian Society for Cell Biology, 15(1), 161-165.

- Takhshid, M. A., Tavasuli, A. R., Heidary, Y., Keshavarz, M., \& Kargar, H. (2012). Protective effect of vitamins E and $\mathrm{C}$ on endosulfan-induced reproductive toxicity in male rats. Iranian journal of medical sciences, 37(3), 173.

- Vieira, A. E., Repeke, C. E., Junior, S. d. B. F., Colavite, P. M., Biguetti, C. C., Oliveira, R. C., . . . Garlet, G. P. (2015). Intramembranous bone healing process subsequent to tooth extraction in mice: micro-computed tomography, histomorphometric and molecular characterization. PloS one, 10(5), e0128021.

- Visweswaran, B., \& Krishnamoorthy, G. (2012). Oxidative stress by tartrazine in the testis of Wistar rats. Journal of Pharmacy and Biological Sciences, 2(3), 44-49.

- Wong, S. K., Mohamad, N.-V., Ibrahim, N. I., Chin, K.-Y., Shuid, A. N., \& Ima-Nirwana, S. (2019). The Molecular Mechanism of Vitamin E as a Bone-Protecting Agent: A Review on Current Evidence. International journal of molecular sciences, 20(6), 1453.

- $\quad$ Xu, W., He, P., He, S., Cui, P., Mi, Y., Yang, Y., Zhou, S. (2018). Gamma-tocotrienol stimulates the proliferation, differentiation, and mineralization in osteoblastic MC3T3E1 cells. Journal of Chemistry, 2018. 